\title{
Gamma-ray burst positions from the ASM on RXTE
}

\begin{abstract}
H.V. Bradt and D.A. Smith
Received March 16; accepted May 10, 1999

Abstract. The RXTE/ASM has detected and positioned 14 confirmed GRB bursts (at this writing, Jan. 1999) including six whose positions were communicated to the community 2 to 32 hours after the burst. Two of these latter bursts led to measurements of optical red shifts but one, despite an easily detected X-ray afterglow, produced no detectable optical or radio afterglow.
\end{abstract}

Department of Physics \& Center for Space Research, Massachusetts Institute of Technology, Cambridge, MA 02139-4307, U.S.A.

the case of GRB, one may have only one sighting. This requires one to impose a higher threshold for detection.

In addition to "position data" that yields intensities and locations of a source based on 90-s integrations, the ASM records "time-series data" in $1 / 8 \mathrm{~s}$ time bins for each of the three energy channels of each of the three detectors. If a new source is detected in the position data of a given
Key words: gamma-ray bursts

\section{ASM capabilities}

The All-Sky Monitor on RXTE (Levine et al. 1996) has the capability to locate Gamma-Ray Bursts (GRBs) to within a few arcminutes in two dimensions. This can occur if the burst falls within the $\sim 10^{\circ}$ by $\sim 50^{\circ}$ parallelogram on the sky which is viewed simultaneously by the two azimuthal shadow cameras of the ASM (see Levine et al. 1996). The solid angle for such detections is actually somewhat larger in some cases because the burst intensity (or that of its immediate afterglow) may remain above ASM threshold as the ASM steps to its next celestial position, i.e. to its next "dwell". This will sometimes bring the burst into the field of view of a camera that had not yet detected it. The ASM takes data for $\sim 40 \%$ of the orbital time.

It is more probable that the burst will fall within the FOV of only one of the three shadow cameras, each with a field of view of $\sim 10^{\circ} \times \sim 100^{\circ}$. In the case of a detection in only one collimator, the error region will be a few arcminutes wide and a few degrees long.

The RXTE does not carry a dedicated GRB detector, and the ASM detects only the X-ray portion of GRB spectra, $1.5-12 \mathrm{keV}$. It is therefore difficult to distinguish a rapid X-ray transient from a GRB. Our original method for securely identifying a new transient was to wait until several sightings confirmed the detection. This would distinguish a genuine transient from background events. In

Send offprint requests to: H.V. Bradt ASM dwell, the presence of rapid variability in the corresponding time-series data improves the likelihood that the source is a GRB.

\section{ASM burst detections}

The RXTE/ASM has detected and positioned 14 GRB since Feb. 1996. Each of the 14 has been confirmed as a burst with detections from GRB detectors on one or more other satellites. Seven of these were detected in two of the ASM shadow cameras, thus yielding positions accurate to a few arcmintues in two dimensions. Of the 14, eight were located in searches of archived data. One of these (GRB 961216) was near the edge of the ASM camera field of view and thus led to a large (and uncertain) error region. The other 7 archival detections and positions are reported in Smith et al. (1999) together with detections and positions of these events from other satellites. They are GRB 960416, 960529, 960727, 961002, 961019, 961029, and 961230 .

Beginning in August 1997, bursts detected by the ASM were analyzed and positions were reported in near real time. Six events have been so reported at times ranging from 2 to 32 hours after the burst. The error regions reported by us and by others for these six bursts are shown in Fig. 1. Notable among these are (1) GRB 970828 (Remillard et al. 1997) which had an easily detected X-ray afterglow but no optical or radio signal (Groot et al. 1998), (2) GRB 980703 (Levine et al. 1998) which led to a radio/optical transient with a redshift $z=0.9660$ (Djorgovski et al. 1998), and (3) GRB 981220 (Smith 1998b) which led to the discovery of a highly variable radio source as a possible counterpart candidate (Galama et al. 1998; Frail \& Kulkarni 1998). The radio source was 


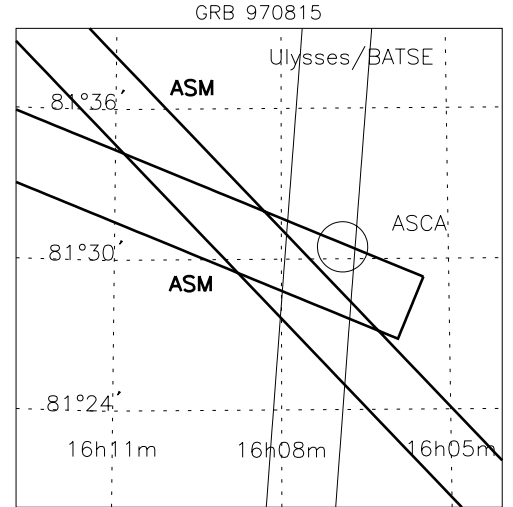

GRB 971214

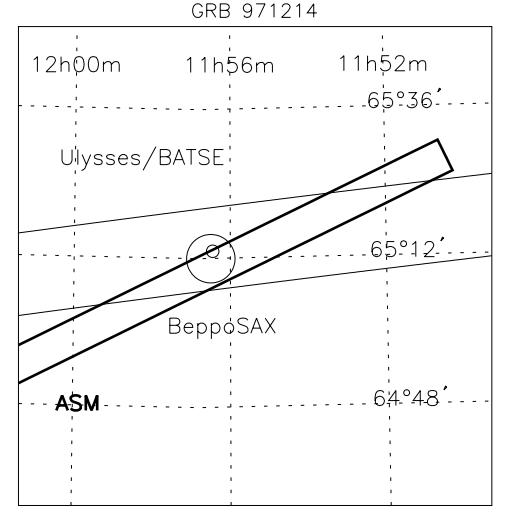

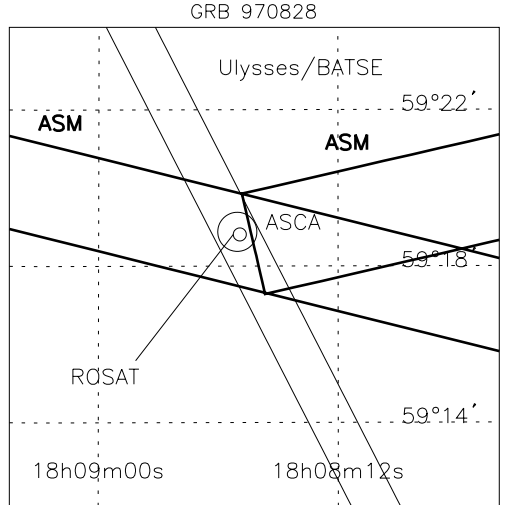

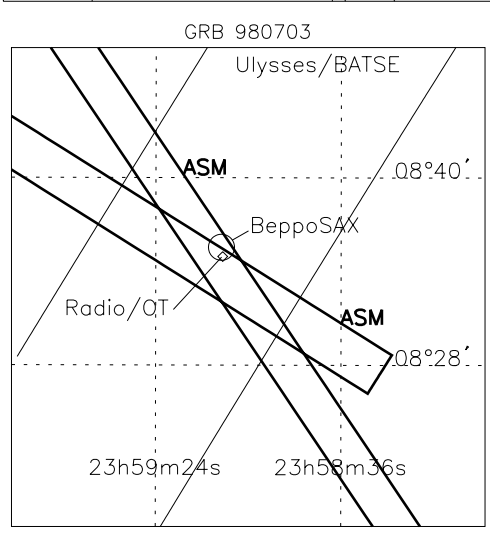

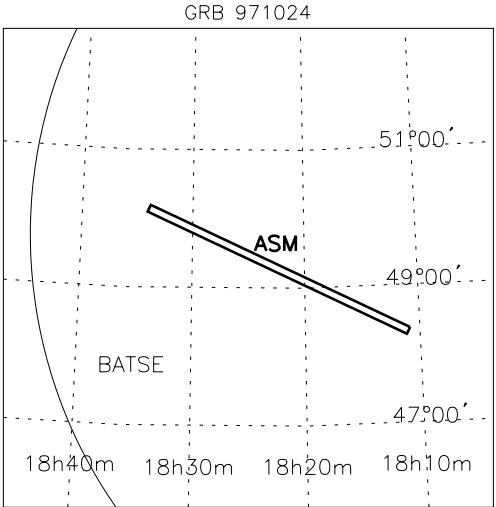

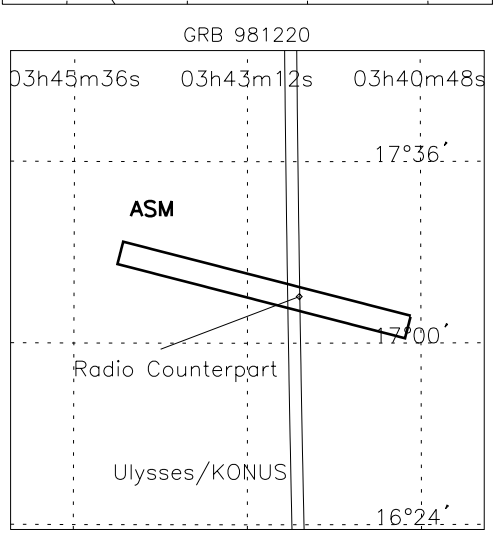

Fig. 1. ASM Positions of the six bursts reported in near real time by the ASM group together with refined BATSE and Interplanetary Network positions from other groups as reported or referenced in Smith et al. (1999)

associated with a faint galaxy at $R=26.4$ (Bloom et al. 1999). The GRB 971214 led to a redshift of $z=$ 3.42 but this result came primarily through the precise two-dimensional positions from SAX (Heise et al. 1997; Antonelli et al. 1997). The ASM results and associated studies are reported or referenced in Smith et al. (1999).

\section{New rapid GRB position GCN alerts}

The capability of the ASM system to produce rapid positions of GRB is improving steadily. We have just initiated (Jan. 1999) automatic transmissions to the GCN when the ASM detects a new transient in two different collimators with sufficient signal strength to rule out noise signals with high confidence. These events can be either a GRB or a new transient, and they will have position precision of a few arcminutes in two dimensions. The trigger criteria would have picked up 6 of the 7 two-collimator burst detections recorded since 1996. In addition, it would also have picked up five new X-ray transients in the past year. For example, a preliminary version of this system detected the bright black hole transient XTE J1550-564 at an intensity of only $70 \mathrm{mCrab}$, very early in its rise from anonymity on 7 Sep. 1998 (Smith 1998a). The position notices are now automatically transmitted to the GCN within 5-60 minutes of the burst arrival time under normal operating conditions. We expect that about 1 to 2 GRB events per year will satisfy the trigger criteria.
Acknowledgements. The entire ASM team at M.I.T. has contributed to this work. We are grateful for the GRB groups of BATSE, Ulysses, and Konus for providing refined GRB positions for inclusion in Smith et al. (1999), some of which are reproduced herein in Fig. 1. Support for the ASM work was provided in part by NASA Contract NAS5-30612.

\section{References}

Antonelli A., et al., 1997, IAU Circ. 6792

Bloom J., Djorgovski S., Kulkarni S., Brauher J., Frail D., Goodrich R., Chaffee F., 1999, GCN Circ. 196

Djorgovski S., Kulkarni S., Bloom J., Goodrich R., Frail D., Piro L., Palazzi E., 1998, ApJ 508, L17

Frail D., Kulkarni S., 1998, GCN Circ. 170

Galama T., Vreeswijk P., van Paradijs J., Kouveliotou C., Strom R., de Bruyn G., 1998, GCN Circ. 168

Groot P., et al., 1998, ApJ 493, L27

Heise J., et al., 1997, IAU Circ. 6787

Levine A., Bradt H., Cui W., Jernigan J.G., Morgan E., Remillard R., Shirey R., Smith D.A., 1996, ApJ 469, L33

Levine A., Morgan E., Muno M., 1998, IAU Circ. 6727

Remillard R., Wood A., Smith D.A., Levine A., 1997, IAU Circ. 6726

Smith D.A., 1998a, IAU Circ. 7008

Smith D.A., 1998b, GCN Circ. 159

Smith D.A., et al., 1999, ApJ (to be submitted) 